\title{
LA EVALUACIÓN DE POLÍTICAS PÚBLICAS EN ESPAÑA: APRENDIZAJE Y PRÁCTICA INSTITUCIONAL
}

Núm. 1 (2013), pp. 31-53.

José María Labeaga Azcona ${ }^{1}$. César Muñoz Martínez²

Recibido: Agosto, 2013

Aceptado: Octubre, 2013

JEL Clasif: $\mathrm{H}_{49}, \mathrm{H}_{5} \mathrm{O}$

\footnotetext{
${ }^{1}$ Department of Economic Analysis in the Universidad Nacional de Educación a Distancia (Madrid.España). jlabeaga@cee.uned.es.

2 Assistant Lecture at the Department of Applied Economics in the UNED. (Madrid.España). cmunoz@cee.uned.es.
} 


\section{Resumen}

La evaluación de políticas públicas en un tema fundamental en cualquier país democrático. En el caso de España esta importancia queda reflejada en varios textos legales como la Constitución Española o la Ley General Presupuestaria. El reconocimiento de dicha importancia no debe ser un fin sino un principio. Los responsables políticos se han encargado de forma continuada de realizar el control del gasto público de acuerdo a los criterios que dicta la normativa citada, pero la evaluación de impacto no ha tenido hasta el momento el papel que la misma parece conferirle. Es, por tanto, necesario dar un paso más al control legal o formal e incluir el punto de vista económico en la evaluación de forma que se constituya dentro del sector público y de forma natural como un juicio a sus intervenciones de acuerdo a los resultados y a los impactos que tengan así como a las necesidades que satisfagan, siempre teniendo en cuenta que las decisiones se adoptan con unos objetivos. Habiéndose avanzado recientemente tanto en el diseño institucional como en algunas aplicaciones, queda mucho camino por recorrer para que la evaluación constituya un verdadero instrumento de apoyo a la toma de decisiones en España. Y para recorrer bien ese camino es necesario primero contar con la transparencia del sector público. Proporcionar la información adecuada es un primer paso necesario no suficiente para cumplir con el principio de transparencia que ha de guiar la actuación de todo decisor de lo público. La descripción de la situación actual y algunas propuestas de desarrollo futuro han tratado de completar una visión tanto del estado actual de la situación en España cuanto de las potencialidades que algunos métodos e instrumentos proporcionan.

Palabras Clave: Políticas públicas; transparencia; métodos de evaluación.

\section{Abstract}

Public policy evaluation is a fundamental issue in any democratic country. Public authorities tried to give this range to evaluation both in the Spanish Constitution as well as in the General Budgetary Law. This acknowledgement should be the beginning of practice and transparency in using public funds. However, impact evaluation is far from getting its expectations. Ex-ante or ex-post auditing is a general and wide recognized practice but impact evaluation had not any effect in the past on the design or change of public policies. We aim in this paper for the necessary use of evaluation as a further step to legal or formal controls in a way such that it should be part of a natural activity in the future of our public sector. Since public policies are implemented according to pre-defined objectives, we should worry about their fulfillment. Spain has institutional design able to conduct impact evaluation so, these institutions need the support of politicians. The complete use of public policy evaluation constitutes a necessary condition for the transparency in the action of the public responsible to 
be true. Moreover, public policy evaluation could constitute an effective instrument in the decision making process. Another necessary condition is to put all the information available for citizens and analysts. We provide in this paper a descriptive study of the current situation of public policy evaluation in Spain as well as some possibilities at hand for giving a more important role to it in the future.

Key Words:Public policies; transparency; evaluation methods . 


\section{Introducción.}

La evaluación de políticas públicas en un tema fundamental en cualquier país democrático. En el caso de España esta importancia queda reflejada en varios textos legales. Así, la Constitución Española en su artículo 31.2 dice: "El gasto público realizará una asignación equitativa de los recursos públicos y su programación y ejecución responderán a los criterios de eficiencia y economía”. De esta forma establece los criterios por los cuales los responsables políticos habrán de asignar los recursos, si bien controlar o contrastar que se asignan de acuerdo a dichos criterios es una cuestión de suma importancia que solo podrá hacerse si se evalúa su cumplimiento tras llevar a cabo la misma.

Por otro lado, la Ley General Presupuestaria, en su artículo 72, establece: “... encomendando al Ministerio de Hacienda, en colaboración con los distintos centros gestores de gastos, el impulso y la coordinación de la evaluación continuada de las políticas de gasto, con la finalidad de que las mismas alcancen sus objetivos estratégicos y el impacto socioeconómico que pretenden”. De una u otra forma y con unos procedimientos u otros, los responsables políticos se han encargado de forma continuada de realizar el control del gasto público de acuerdo a los criterios que dicta la normativa3.

Sin embargo no fue hasta 2006 cuando se creó la Agencia Estatal de Evaluación de las Políticas Públicas y la Calidad de los Servicios (AEVAL), lo que constituyó un reconocimiento expreso de la importancia de dar un paso más desde el control hasta la verdadera evaluación, ya que el objetivo principal de la AEVAL es: “... la promoción y realización de evaluaciones de las políticas y programas públicos cuya gestión corresponde a la Administración General del Estado, favoreciendo el uso racional de los recursos públicos y el impulso de la gestión de la calidad de los servicios”. Este reconocimiento podría, en nuestra opinión, haber enfatizado algo tan importante como el cumplimiento de objetivos estratégicos o la evaluación de impacto. Por tanto, parece una vez más que la normativa prima, por el momento, la importancia de aspectos legales o formales y faltaría dar un paso más en el sentido de incluir el punto de vista

\footnotetext{
${ }^{3}$ Recientemente disponemos de abundante evidencia para aseverar que estas formas de control no han funcionado como se esperaba de ellas y, en algunos casos, no constituyen el procedimiento más adecuado para valorar la mejor asignación de los recursos públicos. Con tanta evidencia de su mal funcionamiento cabría dar el beneficio de la duda a procedimientos nuevos que han mostrado eficacia en otros países.
} 
económico en la evaluación de forma que la misma se constituyera de forma natural como un juicio a sus intervenciones de acuerdo a los resultados y a los impactos que tengan así como a las necesidades que satisfagan. Dicho de otra forma, debiera convertirse en un instrumento sistemático que proporcione evidencia rigurosa de forma que permita informar el proceso de toma de decisiones y ser utilizada como contraste del cumplimiento de objetivos o de la corrección de medidas futuras.

En este artículo se pretende poner de manifiesto la importancia de la evaluación de las políticas públicas, por cualquiera de los procedimiento, ex-ante o ex-post, con cualquier metodología y con el objetivo de valorar si los decisores públicos están llevando a la práctica las mejores medidas y cumpliendo con los objetivos prefijados en las mismas. Se pondrá de manifiesto que con esta tarea se fomenta el aprendizaje por parte de los responsables públicos para la mejora de sus decisiones -que implicará la mejora de las políticas propuestas- y se hará un repaso de la experiencia institucional en la práctica de la evaluación, fundamentalmente en las instituciones públicas del Gobierno Español. Para ilustrar el estado de la cuestión en nuestro país también se realiza un repaso de la práctica de la evaluación en diferentes Centros Directivos de nuestro Ministerio de Hacienda y Administraciones Públicas, en organismos dependientes de otros ministerios y, en particular, la Agencia de Evaluación de Políticas Públicas y Calidad de los Servicios y a nivel superior en instancias de la Unión Europea. Para concluir, se exponen los principales problemas a los que se enfrenta la evaluación en el momento actual y se proponen algunas vías de mejora.

\section{Algunos requerimientos previos para una correcta evaluación de las políticas públicas.}

\section{Fuentes de información.}

Podemos definir la evaluación de políticas públicas como un área de investigación aplicada cuya finalidad es valorar la utilidad y bondad de la intervención pública mediante la aplicación de una o varias técnicas de uso común en ciencias sociales. La Información es clave en la actividad de cualquier organización (pública o privada) para realizar las tareas de planificación, ejecución y control. Como no podía ser de otra forma, la información es también un requerimiento absolutamente esencial para la evaluación de resultados. En este sentido, el sector económico ha sido y es pionero en 
sistemas de información. Basta mirar los medios informativos para darnos cuenta que podemos acceder a datos del sistema financiero en tiempo real a través de la red, tenemos datos de mercados accesibles en todo momento a través de diversos sistemas o se proporcionan indicadores económicos continuamente con el fin que los agentes puedan tomar sus decisiones con el máximo conjunto de conocimientos posible con el objetivo fundamental de reducir la incertidumbre asociada a dichas decisiones. Un segundo requisito para llevar a cabo la tarea de evaluación correctamente es utilizar herramientas disponibles que gestionen la información de manera automática y, por tanto, agilicen el proceso de generación de conocimiento. Y como tercer requisito previo se ha de aplicar las técnicas de forma adecuada al programa o política concretos y a los objetivos de la evaluación de dicho programa o política.

Los dos tipos de información que actualmente se utilizan con mayor frecuencia para realizar evaluaciones son datos de encuestas y datos de registros. La utilización de encuestas ha sido el recurso habitual por cuanto los datos de registros bien no estaban adecuadamente recopilados bien no era posible su utilización por causas diversas. Las encuestas tienen la bondad de la oportunidad de su diseño y de la riqueza de su información y el inconveniente de que se trata de datos que sólo se recopilan con la periodicidad definida en su diseño. Los registros están definidos por las necesidades propias de las instituciones que los generan o de los usuarios, suelen ser menos ricos en información pero se recopilan continuamente en el tiempo y, por tanto, no sufren del problema de obsolescencia de las encuestas. Para el tratamiento de los datos, en muchos casos de una cantidad ingente de observaciones, día a día se desarrollan mejores sistemas de proceso que permiten disponer de forma más ordenada, sistemática y rápida de la información contenida en los mismos. Pero además, las Administraciones Públicas (AA. PP.) se han dotado de sistemas de información que permiten realizar evaluación y control de forma mucho más rápida, han mejorado la recogida y proceso de los datos necesarios y han mejorado sustancialmente la calidad de la información económico-financiera que proporcionan. Todas estas razones actúan de forma conjunta y contribuyen a dar un impulso definitivo en los últimos años a esta área, que primero se constituyó como área pionera de investigación, y que más tarde ha pasado a ser un área fundamental de actuación. 


\section{Momento de la evaluación: Enfoques.}

El enfoque tradicional de la evaluación de políticas ha estado centrado en la fase de decisión relacionada con su aprobación, es decir, con la evaluación ex-ante. En muchos casos, la literatura académica ha denominado a esta forma de valoración de proyectos o reformas potenciales, simulación cuando se trataba de evaluaciones con carácter macroeconómico o microsimulación en el caso microeconómico. Este enfoque primario se ha ido generalizando y adaptando para aplicarlo a programas y a políticas públicas que han pasado a la fase de implementación -se trata de evaluación ex-post o evaluación de impacto-.

La evaluación ex-ante se realiza en el momento previo a la puesta en práctica de las políticas públicas, normalmente consiste en una valoración de la conveniencia de la intervención y en una primera estimación de sus posibles efectos. Este tipo de evaluación se lleva a cabo, generalmente, por la necesidad de disponer de información para discernir entre diferentes políticas sobre la base de los objetivos que con las mismas se pretendan alcanzar.

Las evaluaciones que se realizan tras la puesta en práctica de una reforma, un proyecto o una política se denominan evaluaciones de impacto o ex-post. Por supuesto, si se ha iniciado la reforma o proyecto aunque no haya concluido se pueden llevar a cabo evaluaciones de impacto intermedias que suelen utilizarse para tomar decisiones acerca de la oportunidad de continuar o retirar dicha medida.

Hemos de ser conscientes de que ambos tipos de evaluación son complementarias, y que en la mayoría de los supuestos es conveniente realizar ambos procesos para hacer cumplir la doble finalidad de la evaluación: aprender de la experiencia, extrayendo lecciones que puedan aplicarse a la mejora del diseño y la puesta en práctica de políticas públicas; y rendir cuentas a los ciudadanos que, con carácter general, han sido los que han aportado los recursos utilizados, Feinstein (2007).

Cabe destacar que existen numerosas ocasiones en las que no se realiza ningún tipo de evaluación, en el caso de las evaluaciones ex-ante sí existe apremio por aprobar políticas como señales o indicaciones de que se está haciendo algo en relación a un problema que la población percibe como importante. En el supuesto de las evaluaciones ex-post no siempre se llevan a cabo porque se suelen entender como una 
forma de amenaza para el individuo o institución evaluada, por lo que no se dan los incentivos para que se lleve a cabo.

\section{Dificultad en la definición del objeto a evaluar.}

Otro aspecto importante del proceso de evaluación en el caso de tratarse de evaluar el impacto es conocer y definir bien los objetivos que se persiguen con la política que se pone en marcha. Unos objetivos bien definidos permiten identificar la información que va a resultar necesaria para realizar la evaluación. En algunos casos los objetivos pueden ser muy específicos pero, por regla general se habrán de identificar objetivos con carácter exhaustivo porque es posible que la política tenga efectos diversos.

Estas etapas previas son relevantes porque la evaluación podría quedar invalidada si no se define con carácter previo de forma correcta la población (muestra) y las variables que se han de tener en cuenta en el proceso posterior. Por ejemplo, podemos pensar en una reforma en el mercado de trabajo que se pretende tenga efectos sobre determinados colectivos. Las variables socio-demográficas que definen a dichos colectivos son fundamentales para definir las poblaciones de las que se han de recolectar las muestras para que resulten útiles en el proceso de evaluación posterior. A las dificultades inherentes a la evaluación de programas o políticas que se podría realizar en el sector privado, se unen, en el caso del sector público valoraciones sobre costes o precios cuya definición no es tan clara porque podemos comenzar a introducir conceptos como coste social o beneficio social de determinadas actuaciones que, con carácter general, resultan de difícil valoración pero que es importante tener presente. Esto afecta, asimismo, a la definición de los objetivos y los impactos de la política como consecuencia de que debemos definir funciones objetivo que tengan en cuenta todas las consecuencias de la misma.

\section{Métodos: ¿Cómo evaluar?.}

Como afirma Feinstein (2007) la evaluación de políticas públicas no se limita a preconizar la utilización de un solo método, no existe ningún consenso sobre lo que en cada área de conocimiento se consideran los métodos más adecuados, que en general ha de ser fijado sobre la base de numerosos factores como los datos disponibles, la política que se evalúa, los resultados que se pretende obtener, etc. 
La valoración de las funciones públicas mediante los criterios de eficacia y eficiencia nos permite discernir una primera clasificación teórica y generalista sobre los métodos de evaluación.

Si el proceso evaluador se centra en analizar el grado de cumplimiento de unos objetivos o efectos previstos por una determinada acción política, se utiliza lo que Vedüng (1993) define como modelos de efectividad o análisis de eficacia. Si introducimos aspectos económicos, hemos de añadir a la efectividad el coste de la intervención, por lo que realizaremos análisis de eficiencia. De otro modo, podemos focalizar la evaluación en el análisis de los mecanismos o instrumentos formales a través de los cuáles se han intentado implementar los objetivos, en este caso emplearemos los denominados modelos procedimentales ${ }^{4}$.

En general, la eficacia suele ser más difícil de medir debido a la naturaleza no lucrativa de las instituciones públicas que obliga a introducir el beneficio social en la medición de sus objetivos y a observar la interrelación entre distintas acciones políticas. Para cuantificar, de forma aproximada, los objetivos a cumplir por la intervención se suele emplear como parámetros de evaluación los denominados indicadores5.

Aunque los indicadores se emplean también en los modelos de eficiencia, resulta más común utilizarlos en los análisis de eficacia ${ }^{6}$. Los indicadores cubren una función descriptiva, sistematizando una información que se puede recopilar mediante encuestas o registros y que permite aproximar el grado de cumplimento de los objetivos a evaluar.

Algunos autores aconsejan el uso de indicadores de gestión en la evaluación de la eficiencia, López Hernández y Ortiz Rodríguez (2004) o la utilización de los mismos para realizar análisis coste-eficacia, Albi (1992).

\footnotetext{
${ }^{4}$ Este modelo es el que se ha seguido tradicionalmente en el sector público en España. aunque en la actualidad sigue siendo el modelo dominante existen algunas excepciones. Por ejemplo, en el ámbito universitario se ha impuesto lo que podríamos denominar modelo profesional en el que son los propios compañeros (en un proceso ciego, a veces doble ciego) los que validan los resultados ex - ante (proyectos de investigación) o ex - post (resultados o la docencia).

5 Este instrumento tiene también su plasmación normativa en el artículo 35.4 de la LGP, que establece lo siguiente: "La comprobación del grado de cumplimiento de un programa presupuestario se efectuará en función de los resultados cuando éstos sean mensurables e identificables. Cuando los resultados no sean mensurables la efectividad del programa se hará mediante indicadores que permitan su medición indirecta".

${ }^{6}$ Un ejemplo de elaboración de indicadores de eficacia fue el estudio realizado en Suecia por los autores P.Arvidson, J. Carlsson y A.T. Malm para el Departamento de Policía.
} 
La técnica más empleada para la medición de la eficiencia de un determinado programa político es el análisis coste-beneficio; que consiste en la actualización de la corriente de costes y beneficios de un proyecto, mediante la aplicación de una tasa de descuento, para calcular el VAN social del mismo. Este método es utilizado por ejemplo en la evaluación de proyectos públicos de inversión en infraestructuras de transporte7 ${ }^{7}$.

La dificultad de asignar probabilidades a los flujos de un determinado proyecto, el cálculo de las externalidades y la elección de una tasa de descuento plantea algunos problemas de aplicación en esta técnica de evaluación; por lo que en los últimos años han proliferado otro tipo de estudios relacionados con la minimización de los inputs, sin alterar la calidad de un servicio, o la maximización de los outputs, con vistas a mejorar los procesos o el uso de los recursos, Trillo del Pozo (2002). Este enfoque trata de evaluar la eficiencia de los programas o políticas sobre la base de la productividad, comparando un grupo determinado de unidades de producción pública con una función de producción que define la frontera de posibilidades de producción del mismo. Para hallar la frontera de referencia se pueden utilizar varios tipos de modelos, denominados modelos de fronteras.

En estos modelos podemos distinguir dos aproximaciones; las paramétricas donde la obtención de la frontera se realiza con mínimos cuadrados o con funciones de máxima verosimilitud; y las no paramétricas donde destacan dos técnicas, el Data Envelopment Analysis (DEA) y el Free Disposal Hull (FDH).

Entre los métodos más novedosos que actualmente se están utilizando -y proponiendo- pero que se mantienen fundamentalmente a nivel de investigación teórica $^{8}$ y empírica9, podemos hablar de la evaluación mediante métodos observacionales -cuasi-experimentos-. Estos métodos desarrollados en el campo de la inferencia causal, permiten identificar efectos causales de un programa público a través de la estimación de qué hubiera pasado si no se hubiese implementado una determinada intervención, Galiani y Gasparini (2012).

Para su aplicación es conveniente definir correctamente el experimento (muestras de control y de tratamiento), las condiciones del experimento y los contrafactuales con

\footnotetext{
${ }^{7}$ Guía de análisis costes-beneficios de los proyectos de inversión. Comisión Europea, 2003.

8 Peck, L.R. (2013).

9 Hansen,H., Klejnstrup, N.R., and , and Ole Winckler Andersen, O.L. (2013).
} 
los que comparar los resultados de la política si la misma no se hubiese llevado a la práctica. En estos diseños cuasi-experimentales existen distintos métodos para estimar el parámetro objeto de interés en la evaluación. La literatura económica, Imbens (2004) los categoriza en cuatro áreas: modelos de diferencias en diferencias, el método de las variables instrumentales, el método de regresión y los métodos de emparejamiento.

\section{La experiencia en la evaluación de políticas públicas en el sector público: España y la Unión Europea.}

Hasta la puesta en marcha de la AEVAL en enero de 2007, la experiencia en la evaluación de políticas públicas en nuestro país se limitaba a la evaluación de las distintas condiciones establecidas por la UE en los programas de cofinanciación comunitaria $^{10}$ y a la evaluación de los distintos programas de cooperación al desarrollo (Feinsten and Zapico, 2012). Si bien es cierto, que en las políticas educativas y sanitarias ya existían organismos encargados de medir la eficacia de los programas y la calidad de los servicios prestados, como era la ANECA o INECSE en materia educativa o la Agencia de Calidad del SNS en materia sanitaria, Garde Roca( 2006).

El origen de la evaluación en España, íntimamente ligado al cumplimiento de condiciones comunitarias, limitó su impacto a un nivel gestor y operativo de la acción pública (Ruíz Martínez, 2012). Prueba de ello son algunas de las experiencias que repasaremos a continuación y que se han desarrollado en ámbitos más afines al control presupuestario o al carácter fiscalizador.

El Ministerio de Hacienda y Administraciones Públicas.

Por razones de control financiero, presupuestario o auditoría de cuentas, corresponde al Ministerio de Hacienda y Administraciones Públicas realizar tareas que están próximas a la evaluación pero que generalmente han perseguido más realizar un

\footnotetext{
${ }^{10}$ La Dirección General de Fondos Comunitarios, y particularmente dentro de ella la Subdirección General de Programación Territorial y Evaluación de Programas Comunitarios del MHAP, tiene como tarea primordial la evaluación de los programas que utilizan fondos de la UE. Realizan su trabajo mediante una mezcla de colaboración externa y realización interna. Un ejemplo de su labor interna lo constituye la evaluación previa del programa FEDER (2006).
} 
control legal y formal de las actuaciones del sector público que una verdadera evaluación.

Dentro del Ministerio, la Intervención General de la Administración del Estado (IGAE) es el controlador interno básico del gasto público realizado por el gobierno central. Se realizan auditorías y controles internos de una excelente calidad técnica -lo que constituye un claro ejemplo de calidad en la formalidad del control-. El nivel de desarrollo de la evaluación de impacto es bajo, al no ser un organismo con competencias exclusivas en la materia si bien a nuestro juicio los interventores debieran comenzar a adquirir conocimientos en la fase de oposición y su posterior que les permita en un futuro ser capaces de extender el control a lo que constituiría la última fase de escrutinio del destino adecuado de los fondos públicos. Ejemplos del desarrollo del control y de la transparencia se pueden encontrar en su página web ${ }^{11}$, que en los últimos años ha experimentado un desarrollo muy importante.

Otro organismo dentro del MHAP que tiene entre sus tareas la evaluación de los impactos económico - financieros de las acciones que realiza el sector público, es la Dirección General de Presupuestos (DGP), si bien la evaluación realizada la basan fundamentalmente en el encargo que realizan a instituciones de investigación externa o a expertos externos que colaboran con el personal de la DGP. Un ejemplo de estas colaboraciones es el documento de Boscá, Díaz, Domenech, Ferri, Pérez y Puch (2008), un modelo de equilibrio general para la simulación y evaluación de la economía española que tienen colgado en la página web. Existen otros ejemplos de evaluación y propuestas desde el control presupuestario como el estudio realizado por Zapico (2002), que aunque no contiene evaluación específica de ningún programa o política constituye un alegato interno de lo que se debe hacer y cómo se debe hacer pero no se hace.

La Agencia Estatal de Evaluación de las Políticas Públicas y la Calidad de los Servicios.

Tal como se ha puesto de manifiesto en la introducción a este trabajo, la institucionalización ${ }^{12}$ de la evaluación en España no se materializa en sentido estricto

\footnotetext{
${ }^{11}$ www.igae.pap.minhap.gob.es/

12 Entendemos por institucionalización de la evaluación, el concepto extraído de Garde Roca (2006): “ el proceso político por el que se legitima un marco normativo adecuado para el desarrollo de la función de la evaluación, a partir de la estructuración de espacios e instancias de las Administraciones a las que encargar
} 
hasta la creación ${ }^{13}$ en 2006 de la AEVAL, adscrita en la actualidad al Ministerio de Hacienda y Administraciones Públicas.

Este es el organismo encargado de realizar la evaluación y tiene entre sus objetivos:

- Promover la cultura y la práctica de la evaluación, en todas las fases del ciclo de las políticas públicas y atendiendo a las necesidades estratégicas y de gestión.

- Realizar trabajos de evaluación y análisis de programas y políticas públicas.

- Desarrollar metodologías, sistemas de información e indicadores, así como realizar actividades de acreditación y certificación (niveles de excelencia, buenas prácticas).

- Fomentar la calidad de los servicios públicos como compromiso con la ciudadanía, favoreciendo la participación y la transparencia.

- Analizar las actividades y servicios prestados a los ciudadanos por las Agencias Estatales.

La AEVAL realiza evaluaciones de las políticas encargadas anualmente por el Consejo de Ministros, entre las que figura una evaluación anual del grado de aplicación y de éxito de las principales medidas del Programa Nacional de Reformas, que les encarga la Comisión Delegada del Gobierno para Asuntos Económicos.

Para determinar las evaluaciones que ha realizado la AEVAL desde su puesta en funcionamiento en 2007 hasta 2011, podemos remitirnos al proyecto de investigación: "Elaboración de un mapa de la evaluación de políticas y programas públicos en la Administración General del Estado", realizado por la Universidad Carlos III de Madrid $^{14}$. Cabe destacar que en este trabajo de campo realizado se identificaron 71 evaluaciones para el periodo comprendido entre 2007 y 2011.

tal función, ubicándolos en los distintos niveles de gobierno y según las distintas actividades o sectores a evaluar".

${ }^{13}$ Ley 28/2006, de 18 de julio, de Agencias Estatales para la mejora de los servicios públicos.

14 Dirigido por el Catedrático de Ciencia Política y de la Administración, D. Francisco Vanaclocha Bellver, y financiado por la Agencia de Evaluación y Calidad. 
De estas 71, consideradas como "evaluaciones verdaderas"15, treinta cuatro fueron realizadas por al AEVAL; entre ellas se encontraban las siguientes evaluaciones realizadas antes de 2010:

- El sistema de transferencia de tecnología a empresas.

- El sistema general de becas educativas.

- El sistema de gestión colectiva de los derechos establecidos por la política de propiedad intelectual.

- La participación de la Administración General del Estado en el sistema para la autonomía y la atención a la dependencia.

- La política de bonificación y reducción de cuotas de la Seguridad Social.

- Las acciones financiadas con cargo a los Presupuestos Generales del Estado en las áreas de influencia socioeconómica de la Red de Parques Nacionales.

- Los programas de formación de la cooperación al desarrollo.

En el Informe General de Actividad de 2011 de la AEVAL, se mencionan los trabajos realizados en los años 2010 y 2011; en base a los Planes de Trabajo de 2010 y 2011 aprobados en sendas órdenes ${ }^{16}$ del Consejo de Ministros. En el año 2010, se realizaron las siguientes evaluaciones de programas y políticas públicas:

- Plan español de energías renovables 2005-2010;

- Líneas ICO-PYME;

- Programa de ayuda a la reindustrialización;

- Estrategia de salud de ciudadanos paliativos;

- Programa de vacaciones para personas mayores y termalismo social;

\footnotetext{
${ }^{15}$ El concepto de "evaluaciones verdaderas" se asocia a la terminología de Stuffleban y Sinkfield (1985): como procesos evaluativos que producen evidencia científica a partir de una investigación sistemática; que tienen por objeto intervenciones públicas identificables como políticas o planes públicos; que incorporan un juicio de valor sobre la base de criterios de valor explícitos o implícitos; y que proporcionan orientaciones prácticas en relación con la intervención evaluada, Ruíz Martínez (2012).

16 Orden PRE/1965/2010, de 18 de junio de 2010 y Orden TAP/700/2011, de 17 de marzo de 2011.
} 
- La gestión y funcionamiento de las demarcaciones de costas para la protección del dominio público marítimo terrestre.

Mientras que en el año 2011:

- La segunda fase de las líneas ICO-PYME;

- La evaluación sobre las trabas administrativas para la creación de empresas;

- Informe relativo a la eliminación de las posibles duplicidades, solapamientos e ineficiencias en el estado Autonómico.

Además, en 2011, se incorpora por primera vez la posibilidad de realizar Convenios de Colaboración celebrados entre la propia Agencia y diversas CCAA; realizándose las siguientes evaluaciones conjuntas:

- La evaluación externa de la calidad del servicio prestado por ventanilla única para el comercio exterior de canarias (VEXCAN);

- La evaluación del plan de acción integral de la playa de la Palma;

- La evaluación de los programas de selección y formación de la escuela de Administración Regional de Castilla La-Mancha.

En junio de 2012, la Secretaria de Estado de Administraciones Públicas encargó un estudio sobre la actividad que se desarrolla en los servicios centrales de los Ministerios, trabajo que se encuentra en trámite en la actualidad. De otra forma, el Informe General de AEVAL de 2012 también muestra la realización de dos trabajos de explotación de datos en el año referido:

- Análisis de la Política de Saneamiento y Depuración, a partir de los informes encargados a TRAGSA para la evaluación de Confederaciones Hidrográficas;

- Estudio sobre la percepción de los ciudadanos en relación con los resultados de la política educativa (Barómetro del CIS de marzo de 2012)

En cuanto a las evaluaciones que habían sido incluidas en el Plan de Trabajo de 2013 figuran las que se presentan a continuación:

- El Plan de Ahorro y Eficiencia Energética 2008-2012; 
- La Formación dirigida a empleados públicos en la Administración General del Estado;

- El Plan de Medidas para la Mejora de los Servicios de Sanidad Exterior;

- Diseño de una metodología para caracterizar las funciones desarrolladas por los servicios centrales de la AGE;

- Diagnóstico de situación y rediseño del Sistema de Información Administrativa.

Todos los documentos resultantes de sus evaluaciones se pueden consultar en la web $^{17}$ de AEVAL. Resulta frustrante comprobar que ni las políticas evaluadas son las que se esperaría durante los años de funcionamiento de AEVAL ni los informes constituyen ejercicios de evaluación del impacto de dichas políticas. En su favor se podría argumentar que no tiene competencias en evaluación de políticas regionales (las correspondientes a los gobiernos autonómicos) pero ello no es óbice para que podamos calificar su actuación durante estos años como decepcionante.

\section{La Unión Europea.}

La Unión Europea tiene bastante desarrollados los procedimientos de evaluación, que se refieren a los fondos que reciben los países miembros y que se encarga de realizarlas cada país pero que también hace referencia a sus actividades internas. Cuentan con numerosas evaluaciones realizadas en diversos ámbitos y con el fin de no resultar exhaustivo en su enumeración citaremos dos, una constituye un ejemplo de evaluación ex-ante y consistió en la evaluación de la contratación pública con el fin de contribuir a la mejora medio-ambiental (European Comission, 2008); la otra es un ejemplo de evaluación ex-post y consistió en evaluar la asistencia macro-financiera proporcionada por la Unión Europea a Albania (Hiddink, Bolognini, Paczynski, Pot, Shano y de Groot, 2008).

17 www.aeval.es/es/index.html 


\section{Algunas propuestas de evaluación desde instituciones de la AGE.}

Como hemos visto, existen en la AGE, al margen de AEVAL, otras instituciones que incorporan a su funcionamiento diario tareas de evaluación de diferentes políticas. El Instituto de Estudios Fiscales (IEF) es el centro especializado de formación e investigación al servicio de la Hacienda Pública Española. Sus misiones institucionales básicas consisten en impulsar y articular núcleos de investigación y reflexión (propios y externos) que conecten con las necesidades de la Hacienda Pública y de la sociedad civil así como desarrollar servicios de formación de calidad al servicio de las políticas y objetivos estratégicos del MHAP. La evaluación es una actividad transversal que tiene efectos sobre muchas de las líneas de investigación que se desarrollan en el IEF. En este epígrafe se describen algunas actividades que se desarrollan o han desarrollado en el IEF, por lo que se refiere a la evaluación de las políticas tanto del propio MHAP como de otros ministerios, en tanto en cuanto tengan efectos sobre los ingresos y los gastos públicos.

Un primer instrumento de evaluación con el que cuenta el IEF y que se terminó de actualizar a la situación del IRPF en 2009 es un microsimulador de reformas del IRPF (MICROSIM). Cuando se lleva a cabo una reforma impositiva son numerosas las cuestiones que el decisor público tiene que valorar al nivel de evaluación de la misma. Por ejemplo, se puede preguntar si se perderá o ganará recaudación (en el caso de no ser una reforma neutral en términos recaudatorios). Pero también vale la pena preguntarse los efectos que dicha reforma tendrá sobre el bienestar de los contribuyentes, sobre sus nuevos pagos impositivos, sobre la distribución de la renta, sobre determinados colectivos de la población más expuestos a situaciones de pobreza o exclusión, etc. El IEF ha estado permanentemente preocupado por dar respuesta a estas cuestiones y ofrecer dicha respuesta a los responsables dentro del MHAP de decidir sobre el alcance y el contenido de potenciales reformas impositivas. En este sentido, MICROSIM constituye un instrumento que permite valorar ex-ante los impactos de cambios impositivos directos ${ }^{18}$. En el caso que estamos exponiendo, solamente nos preocupamos de los efectos inmediatos puesto que no se incorpora la respuesta de los agentes a dichos cambios. La incorporación, no obstante, solamente

18 Ver Ayala et al., (2004). 
constituye un modelo adicional que actúa con carácter previo a la determinación de la renta tras la reforma, afectando a determinados individuos de la población.

La realización de MICROSIM tiene en cuenta, en un proceso secuencial, los requerimientos citados en relación con la evaluación. A saber, la información disponible, que en este caso está constituida por registros del panel de declarantes de IRPF proporcionado por la Agencia Estatal de Administración Tributaria (AEAT), la función objetivo (la determinación de la renta tras la aplicación de la legislación del IRPF en el momento de la reforma). La definición de los objetivos estará fundamentada en los propósitos del decisor público, pero MICROSIM incorpora rutinas para la evaluación de la recaudación y numerosas medidas de bienestar y redistribución que surgen tras la incorporación de cambios impositivos. Entre los resultados de la simulación se ofrecen una amplia variedad para mejor información de la pertinencia de tomar determinadas decisiones. Por otro lado, si se desea evaluar los impactos de una reforma tras su puesta en práctica, MICROSIM es susceptible de ser utilizado, con la única restricción de valorarlos cambiando la muestra sobre la que se realiza dicha valoración, que habrá de ser necesariamente posterior a la puesta en marcha de los cambios ${ }^{19}$.

De la misma forma que se dispone de un instrumento para valorar los impactos tras cambios en el IRPF, el IEF también ha desarrollado modelo de Microsimulación y comportamiento económico en el análisis de reformas de imposición indirecta (Impuesto sobre el Valor Añadido e Impuestos Especiales), denominado SINDIEF²0.

Instrumentos de esta naturaleza pueden (y deben) utilizarse para valorar impactos de diversa índole, aunque por lo se refiere al IEF el interés básico es valorar impactos distributivos de políticas microeconómicas surgidas tras reformas fiscales, tras cambios en el sistema de subsidios o transferencias, generadas por cambios en los programas de gasto público (sanidad, dependencia, pensiones, etc.), en programas de empleo, pero también impactos distributivos de políticas macroeconómicas (shocks en precios del petróleo o de los alimentos, por ejemplo) o de cambios demográficos que pueden afectar las pensiones, el sistema sanitario u otros.

\footnotetext{
${ }^{19} \mathrm{El}$ instrumento puede ser utilizado por los interesados solicitando acceso a los responsables del mismo en el IEF.

${ }^{20}$ Más información en Sanz et al., (2003).
} 
Otro de los modelos dentro de esta técnica de microsimulación que se ha diseñado en España es el proyecto que está realizando la Fundación Rafael del Pino, en colaboración con el MHAP, el MECC, BBVA, la Universidad de Valencia y la Fundación de Economía Analítica con el objetivo de desarrollar modelos económicos para analizar, diseñar y evaluar las políticas públicas destinadas a promover la estabilidad macroeconómica, el pleno empleo, la cohesión regional y la convergencia con los países del entorno.

\section{Conclusiones.}

En este trabajo se ha tratado de poner de manifiesto la importancia de la evaluación de las políticas públicas, por cualquiera de los procedimiento, ex-ante o ex-post y con cualquier metodología. Es bien cierto que realizar adecuadamente esta tarea fomenta el aprendizaje por parte de los responsables públicos para la mejora de sus decisiones. Por otra parte se ha realizado un repaso de la experiencia institucional en la práctica de la evaluación, fundamentalmente en las instituciones públicas del Gobierno Español y, en particular, en el MHAP. También se han expuesto los objetivos y tareas de la AEVAL que se encarga de la evaluación de impacto de las políticas públicas en España desde 2006. Finalmente, se han presentado instrumentos que permiten la evaluación ex-ante y ex-post de políticas públicas que afectan al ingreso y al gasto público, que se están desarrollando en el Instituto de Estudios Fiscales. Creemos que con carácter previo a la puesta en marcha de cambios, la información que estos instrumentos pueden proporcionar a los decisores de las políticas pública es muy útil para valorar los efectos de las mismas. De la misma forma, estos instrumentos se pueden utilizar para evaluar ex-post si se han cumplido los objetivos para los que se diseñaron las políticas.

Por supuesto, el trabajo se ha presentado a nivel muy descriptivo y sin entrar en los detalles técnicos que la correcta evaluación de las políticas públicas requiere. Nuestra opinión es que se están comenzando a dar pasos importantes en esta área aunque el diseño institucional y la preparación tanto del capital humano como del capital tecnológico de las AA. PP: deben permitir en el futuro avanzar en el cumplimiento de lo que nuestra legislación reconoce como derechos de los ciudadanos que no es más que ofrecer la información y el servicio prestado con el máximo grado de transparencia y eficiencia para que la tarea se pueda valorar correctamente y los ciudadanos puedan tomar sus decisiones con el máximo volumen de información posible. 
Por otra parte, creemos que la actual coyuntura de austeridad presupuestaria no debe ser freno para el desarrollo de actuaciones en el marco de la evaluación de políticas públicas; puesto que ésta debe entenderse como una inversión y no como un coste. 


\section{Referencias Bibliográficas}

Agencia Estatal de Evaluación de las Políticas Públicas y la Calidad de los Servicios (2012). Informe General de Actividad 2012. Junio 2013, Ministerio de Hacienda y Administraciones Públicas. 100 p.

Agencia Estatal de Evaluación de las Políticas Públicas y la Calidad de los Servicios (2011). Informe General de Actividad 2011. Junio 2012, Ministerio de Hacienda y Administraciones Públicas. 97 p.

Agencia Estatal de Evaluación de las Políticas Públicas y la Calidad de los Servicios (2010). Informe General de Actividad 2010., Ministerio de Política Territorial y Administraciones Públicas. 108 p.

Albi, E. (1992), "Evaluación de la eficiencia pública. El control de la eficiencia del sector público”, Hacienda Pública Española, no 120-21, 299-316.

Arvidson, P.; Carlsson, J., y Malm, A.T.: Accounting and Control in Police Force, 13 th. Annual Congress of the European Accounting Association (EAA), Budapest, 1990.

Ayala, 1.; Onrubia, J. et Ruiz-Huerta, J. (2004): "Modelos de microsimulación: aplicaciones a partir del Panel de Declarantes por IRPF del Instituto de Estudios Fiscales”, Cuadernos Económicos ICE, nº 68, 111-137.

Blanco, A., P. Jurado, M. Méndez, E. Montagud, F. Piedras y F. Vilchez (2006), "La evaluación del desempeño en el contexto del liderazgo en el Ministerio de Economía y Hacienda”, DT. Instituto de Estudios Fiscales.

Boscá, J.E., A. Díaz, R. Domenech, J. Ferri, E. Pérez y L. Puch (2008), “A Rational Expectations Model for Simulation and Policy Evaluation of the Spanish Economy”, DGP. DT 2007-04.

Comisión Europea (2003): Guía de análisis costes - beneficios de los proyectos de inversión.

Dirección General de Fondos Comunitarios (2006), "Evaluación previa del programa operativo del Fondo de Cohesión - FEDER”, Documento DGFC. 
European Comission (2008), "Public procurement for a better environment", Comunicación de la Comisión al Parlamento Europeo, al Consejo, al Comité Económico y Social Europeo y al Comité de las Regiones, COM (2008) 400, de 19 de julio de 2008.

Feinstein, Osvaldo (2007), "Evaluación pragmática de políticas públicas”, Información Comercial Española, № 836, 19-31.

Feinsten, O and Zapico, E. (2012): Evaluation of Government Performance and Public Policies in Spain. Independent Evaluation Group, The World Bank. Working Paper Series No 22.

Galiani, S., Gasparini, L. (2012): El impacto distributivo de las políticas sociales. Facultad de Ciencias Económicas, Universidad Nacional de La Plata. Documento de trabajo $\mathrm{N}^{\circ} .130$

Garde Roca, J.A.(2006), "La institucionalización de la evaluación de las políticas públicas en España”, Auditoría Pública nº 39 ,17-26.

Hansen,H., Klejnstrup, N.R., and , and Ole Winckler Andersen, O.L. (2013), "A Comparison of Model-Based and Design-Based Impact Evaluations of Interventions in Developing Countries”, American Journal of Evaluation, 34(3), 320-338

Hiddink, C., A. Bolognini, W. Paczynski, F. Pot, J. Shano y A. de Groot (2008), "Ex post evaluation of MFA operations in Albania”, WP. Ecorys.

Imbens, G. (2004). "Nonparametric estimation of average treatment effects under exogeneity: A review”, Review of Economics and Statistics 86(1), 4-29.

Lopez Hernández, A., Ortiz Rodríguez, D (2004), "Los indicadores de gestión y el control de eficiencia del sector público", Revista española de control externo, Vol. 6, $\mathrm{N}^{\mathrm{O}} 18,189-220$. 
Loscos Fernández. F.J.: Eficiencia, equidad y control democrático: un marco triangular para el análisis de las políticas públicas, en VII Congreso Internacional del CLAD sobre la Reforma del Estado y de la Administración Pública, Lisboa, Portugal, 811 Oct. 2002.

Peck, L.R. (2013), "On Analysis of Symmetrically Predicted Endogenous Subgroups: Part one of a Method Note in Three Parts", American Journal of Evaluation, 34(2), 225-236.

Ruiz Martínez, A (2012), "Panorámica actual de la evaluación de las políticas públicas”, Presupuesto y Gasto Público 68/2012, 13-23.

Sanz, J.F., Romero, D., Castañer, J., Prieto, J., Fernández, J.F., (2003). Microsimulación y comportamiento en el análisis de reformas de imposición indirecta. El simulador de Imposición Indirecta del Instituto de Estudios Fiscales (SINDIEF). Instituto de Estudios Fiscales, Madrid.

Stufflebeam, D.L. y Sinkfield, A.J. (1985): "Systematic Evaluation. A Self-Instructional Guide to Theory and Practice”. Kluwer-Nijhoff. Boston.

Trillo Del Pozo D.: Análisis económico y eficiencia del sector público. VII Congreso Internacional del CLAD sobre la Reforma del Estado y de la Administración Pública; 2002 Oct 8-11. Lisboa: Portugal; 2002

Vedüng, E. (1993), “Utilización de la evaluación”, Revista de Servicios Sociales y Política Social 30, 69-80.

Zapico, E. (2002), "Reformas en la gestión del gasto público en la OCDE: Sugerencias para una nueva Ley General Presupuestaria”, Presupuesto y Gasto Público 30, 111-128.Apellido, Iniciales. \& Apellido, Iniciales. (Año).. 